\title{
BMJ Open Optimised transcranial direct current stimulation (tDCS) for fibromyalgia - targeting the endogenous pain control system: a randomised, double-blind, factorial clinical trial protocol
}

\author{
Luis Castelo-Branco, ${ }^{1}$ Elif Uygur Kucukseymen (D , ${ }^{1}$ Dante Duarte, ${ }^{1}$ \\ Mirret M El-Hagrassy, ${ }^{1}$ Camila Bonin Pinto, ${ }^{1}$ Muhammed Enes Gunduz, \\ Alejandra Cardenas-Rojas (D) , ${ }^{1}$ Kevin Pacheco-Barrios, ${ }^{1}$ Yiling Yang, ${ }^{1}$ \\ Paola Gonzalez-Mego (1D , ${ }^{1}$ Anayali Estudillo-Guerra, ${ }^{1}$ Ludmilla Candido-Santos, ${ }^{1}$ \\ Ines Mesia-Toledo, ${ }^{1}$ Haley Rafferty, ${ }^{1}$ Wolnei Caumo, ${ }^{2}$ Felipe Fregni ${ }^{1}$
}

To cite: Castelo-Branco L, Uygur Kucukseymen E, Duarte D, et al. Optimised transcranial direct current stimulation (tDCS) for fibromyalgiatargeting the endogenous pain control system: a randomised, double-blind, factorial clinical trial protocol. BMJ Open 2019;9:e032710. doi:10.1136/ bmjopen-2019-032710

- Prepublication history and additional material for this paper are available online. To view these files, please visit the journal online (http://dx.doi. org/10.1136/bmjopen-2019032710).

\section{LC-B and EUK are joint first} authors.

Received 13 August 2019 Revised 29 August 2019 Accepted 30 August 2019

Check for updates

(C) Author(s) (or their employer(s)) 2019. Re-use permitted under CC BY-NC. No commercial re-use. See rights and permissions. Published by BMJ.

For numbered affiliations see end of article.

Correspondence to

Dr Felipe Fregni;

fregni.felipe@mgh.harvard.edu

\section{ABSTRACT}

Introduction Fibromyalgia (FM) is a common debilitating condition with limited therapeutic options. Medications have low efficacy and are often associated with adverse effects. Given that FM is associated with a defective endogenous pain control system and central sensitisation, combining interventions such as transcranial direct current stimulation (tDCS) and aerobic exercise (AE) to modulate pain-processing circuits may enhance pain control. Methods and analysis A prospective, randomised (1:1:1:1), placebo-controlled, double-blind, factorial clinical trial will test the hypothesis that optimised tDCS (16 anodal tDCS sessions combined with AE) can restore of the pain endogenous control system. Participants with FM $(n=148)$ will undergo a conditioning exercise period and be randomly allocated to one of four groups: (1) active tDCS and AE, (2) sham tDCS and AE, (3) active tDCS and non-aerobic exercise (nAE) or (4) sham tDCS and nAE. Pain inhibitory activity will be assessed using conditioned pain modulation (CPM) and temporal slow pain summation (TSPS) - primary outcomes. Secondary outcomes will include the following assessments: Transcranial magnetic stimulation and electroencephalography as cortical markers of pain inhibitory control and thalamocortical circuits; secondary clinical outcomes on pain, FM, quality of life, sleep and depression. Finally, the relationship between the two main mechanistic targets in this study - CPM and TSPS — and changes in secondary clinical outcomes will be tested. The change in the primary efficacy endpoint, CPM and TSPS, from baseline to week 4 of stimulation will be tested with a mixed linear model and adjusted for important demographic variables.

Ethics and dissemination This study obeys the Declaration of Helsinki and was approved by the Institutional Review Board (IRB) of Partners Healthcare under the protocol number 2017P002524. Informed consent will be obtained from participants. Study findings will be reported in conferences and peer-reviewed journal publications.

Trial registration number NCT03371225.

\section{Strengths and limitations of this study}

- A sham-controlled, powered clinical trial on a novel low-cost therapy for fibromyalgia.

- Endogenous pain system biomarkers will help reveal the mechanisms of fibromyalgia as well as the interventions.

- This study will inform us on the number of sessions needed to induce significant changes in neuroplasticity reflected in the above mentioned markers.

- The secondary outcomes of this study will evaluate the suitability of the proposed biomarkers to predict treatment response.

- Exclusion of patients with increased risk during exercise may limit the generalisability of the findings.

\section{INTRODUCTION}

Fibromyalgia (FM) affects about $2 \%$ of the world population ${ }^{1}$ and is associated with poor quality of life mainly due to pain, fatigue, sleep disturbances, functional limitations and cognitive impairments. ${ }^{2}$ Current treatments for this challenging complex condition lead to an average annual cost of $\$ 5945$ in insurance claims per patient with FM, more than twice the amount of a typical beneficiary. ${ }^{3}$ The treatment of choice is a multimodal approach that includes selfmanagement strategies, ${ }^{4}$ but there is a large gap between supply and demand as access to such therapies is limited. Consequently, many patients with FM rely on pharmaceuticals such as non-steroidal anti-inflammatory drugs, antidepressants and/or anticonvulsants, which usually do not provide enough symptom relief and are frequently associated with adverse effects. ${ }^{5}$ Therefore, there is an 
urgent need for the development of novel and targeted treatments with fewer side-effects.

\section{Rationale and gap}

Accumulating evidence ${ }^{6-9}$ shows that disturbances in the endogenous pain control system lead to chronic pain. Several neurophysiological ${ }^{10-16}$ and neuroimaging ${ }^{17-21}$ studies showed altered pain processing mechanisms in FM; therefore, therapies that target and modulate the neural circuits involved in pain control are essential to treat FM characteristic chronic widespread pain. Different ways to potentially modulate these circuits include exercise-which has a known evidence-based therapeutic effect on pain in $\mathrm{FM},{ }^{22}$ and non-invasive neuromodulation techniques such as transcranial direct current stimulation (tDCS) - which demonstrably improve several chronic pain conditions. ${ }^{23-28}$ Despite its investigated benefits to treat different pain conditions (typically targeting the primary motor cortex (M1)), tDCS effects in FM have been mixed. ${ }^{29-32}$ Yet, tDCS can be easily coupled to other therapies due to its low-cost and portability, ${ }^{33}$ and such combinations have been superior to either of the therapies alone in other disorders. ${ }^{34-36}$ We have shown in a pilot study with $45 \mathrm{FM}$ subjects that combining exercise and tDCS for FM leads to a significant pain decrease that also shows a different neural signature as compared with each therapy alone (tDCS or exercises) ${ }^{37}$ In this initial study, however, the endogenous pain inhibitory system was not assessed.

Given the extensive data showing that (i) FM has a defective endogenous pain inhibitory system ${ }^{10-16}$ and (ii) exercises ${ }^{38-40}$ and tDCS lead to modulation of this system, ${ }^{314142}$ we then hypothesised that these two neuromodulatory techniques can help restore the endogenous pain inhibitory system in FM. Neurophysiological and clinical assessments including electroencephalography (EEG), transcranial magnetic stimulation (TMS), quantitative sensory testing and questionnaires for pain and quality of life can provide important data to understand how the endogenous pain inhibitory system is then modulated by these two interventions.

\section{Research question and hypothesis}

We therefore aimed to test whether in subjects with FM 16 sessions of M1 anodal tDCS combined with aerobic exercise (AE) decrease temporal slow pain summation (TSPS) and increase conditioned pain modulation (CPM) responses compared with each intervention alone and to sham when assessed on the last day of intervention. We hypothesise that this optimised tDCS plus AE technique will lead to a stronger engagement of the endogenous pain regulatory system, which will ultimately lead to increased pain regulation in patients with FM.

\section{OBJECTIVES}

\section{Primary objective}

- To evaluate the effects of 4 weeks of tDCS plus AE on the endogenous pain regulatory system (assessed by CPM) and central sensitisation (assessed by TSPS) compared to either interventions alone and to no intervention.

\section{Secondary objectives}

- To determine the effect of these interventions on cortical markers of inhibitory control that are also altered in FM, such as intracortical inhibition assessed by TMS, and changes in thalamocortical dysrhythmia (TCD) and event-related desynchronisation (ERD) assessed by EEG.

- To assess whether engagement of the two main targets tested in this study (TSPS and CPM) are associated with the secondary clinical outcomes (i.e., changes in pain outcomes: Brief Pain Inventory, Revised Fibromyalgia Impact Questionnaire).

- To assess EEG changes across groups and their suitability as potential markers of TCD normalisation.

- To determine the number of sessions needed to induce significant changes in markers of the endogenous pain inhibitory system and central sensitisation (CPM and TSPS) and cortical changes (paired pulse TMS and EEG).

\section{METHODS AND ANALYSIS}

\section{Trial design}

This is a single centre 4-arm factorial Randomized Clinical Trial (RCT). Participants will be randomised using a random blocked randomisation sequence generated by a computer software. We used a 1:1:1:1 allocation ratio to active or sham tDCS combined with $\mathrm{AE}$ or non-aerobic exercise (nAE) on the first day of the conditioning exercise programme. The staff member performing randomisation will not be involved in the trial otherwise. Sequentially numbered sealed envelopes will maintain allocation concealment. Investigators providing assessments will be blinded to tDCS but not exercise. Assessors of primary and secondary outcomes (and participants) will be blinded to group allocation (see figure 1 for group allocation).

\section{Study setting}

This is a single-site study, and all procedures will be conducted at the Neuromodulation Center, Spaulding Rehabilitation Hospital. Enrolment start date is 1 May 2019 and expected end date is 31 December 2023.

\section{Eligibility criteria}

We will use broad-based recruitment strategies, including online advertisements, flyers, clinician referrals and so on. All eligible participants must fulfil the inclusion criteria and have none of the exclusion criteria listed in box 1 .

As part of the eligibility criteria, participants will perform a pretraining visit to evaluate if they are comfortable with walking on the treadmill at a self-selected speed at their baseline heart rate (HR) for $30 \mathrm{~min}$. Only subjects comfortable with this task will be randomised. If the subject is unable to walk for 30 min on the treadmill or reports discomfort or any side-effects precluding physical exercise (eg, excessive muscle soreness), they will be 


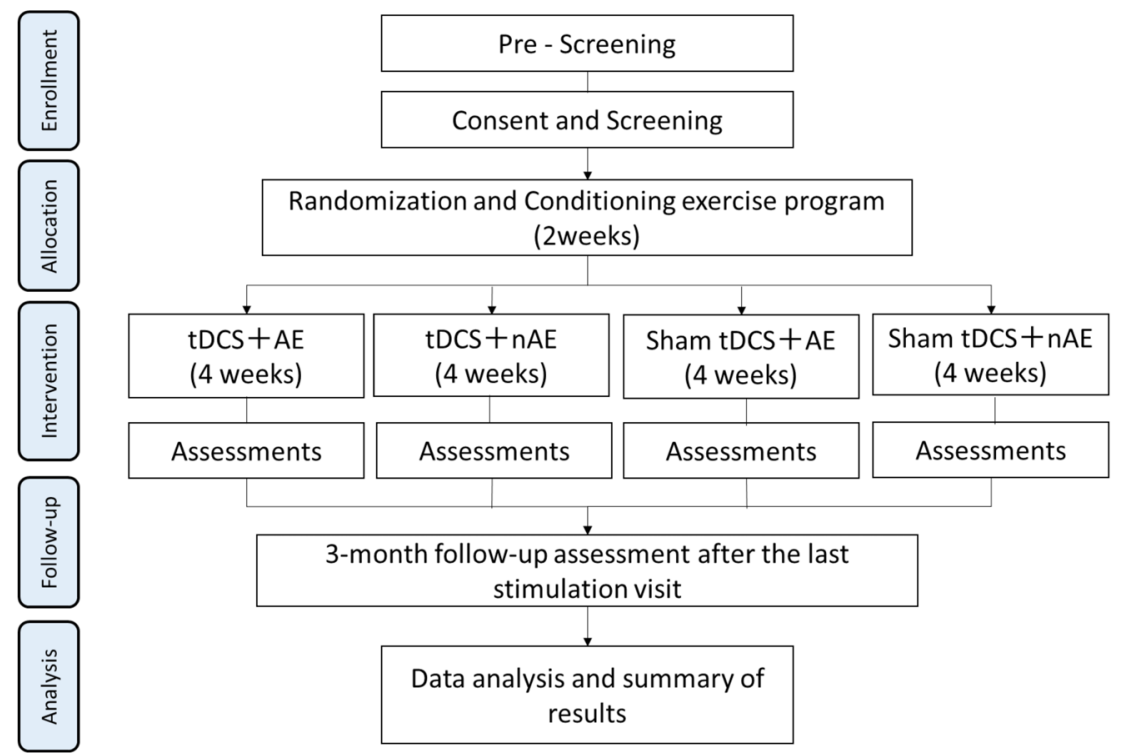

Figure 1 Flowchart of the study based on CONSORT criteria. AE, aerobic exercise; nAE, non-aerobic exercise; tDCS, transcranial direct current stimulation.

screened out. Also, a demographic survey will be taken during the consent visit.

\section{Intervention}

Exercise

Conditioning exercise programme: 6 exercise sessions are divided in 3 days per week over 2 weeks. Duration of sessions will start with $10 \mathrm{~min}$ and increase gradually, ending with a $30 \mathrm{~min}$ session on the last day. The $\mathrm{AE}$ group will walk briskly at $60 \%-70 \%$ of their maximum HR and the nAE group will walk within $5 \%$ of their baseline HR. If a participant on the AE group is unable to progress beyond 15 min at $60 \%-70 \%$ hour max over the initial 2 weeks, they will be screened out of the study. After the conditioning exercise programme, subjects will continue with the intervention part of the protocol. Participants will complete AE or nAE three times a week on non-consecutive days over 4 weeks.

Aerobic exercise ( $A E)$ : Participants will undergo moderate intensity $\mathrm{AE}$ on a treadmill over 30 min (American Heart Association recommendation for adults). HR will be monitored throughout the entire procedure by a sensor. The investigator will sequentially increase the treadmill speed by $0.1 \mathrm{mph}$ every $5 \mathrm{~s}$, until the participant reaches $60 \%-70 \%$ of age-predicted maximal heart rate (HRmax), following the formula HRmax $=208-(0.7 *$ age $)$, as this has been found safe in various conditions. ${ }^{2243-47} \mathrm{AE}$ intensity will be modulated based on the participant's HRmax throughout the session. If the HRmax exceeds $70 \%$, the investigator will decrease treadmill speed by $0.1 \mathrm{mph}$ every 5 s until returning to the $60 \%-70 \%$ HRmax target. If HRmax reaches $80 \%$ or the subject shows any signs of discomfort, the session will be stopped.

Non-aerobic exercise ( $n A E)$ : Participants will walk on the treadmill for 30 min with a workload intensity within $5 \%$ baseline HR, as we used this method in our preliminary study. ${ }^{37}$

As recommended by ACSM guidelines for $\mathrm{AE}$ in patients with FM, the participant will be questioned regarding any respiratory or cardiovascular symptoms on each visit before starting the exercise; we will monitor pain and fatigue levels after the first 5, 15 and $25 \mathrm{~min}$ of exercise using a numeric pain scale. ${ }^{48}$ Additionally, to evaluate adverse effects during $\mathrm{AE}$ or $\mathrm{nAE}$ training, we will record any musculoskeletal symptoms such as pain, muscle strain, muscle soreness, fatigue, dizziness and shortness of breath.

Transcranial direct current stimulation (tDCS)

A $1 \times 1$ low-intensity DC stimulator, the Soterix Medical $1 \times 1$ tDCS-Clinical Trial, will be used with codes corresponding to active or sham stimulation, allowing a double-blinded procedure. Participants will receive 16 tDCS sessions over 4 weeks of treatment. Weeks 1 and 2 will begin with five consecutive days of tDCS followed by weeks 3 and 4 with three alternating days of tDCS. The exercise and the tDCS will be performed simultaneously as explained in figure 2.

Active (anodal) tDCS: During active tDCS, a $2 \mathrm{~mA}$ constant current will be delivered for $20 \mathrm{~min}$ through rubber electrodes encased in $35 \mathrm{~cm}^{2}$ saline-soaked sponges. The anode will be placed over the left primary motor cortex (M1) and the cathode over the contralateral supraorbital area. M1 will be localised using the 10/20 International EEG System (C3-adapted by measuring $5 \mathrm{~cm}$ below the vertex), a reliable method for tDCS. ${ }^{23}$

Sham $t D C S$ : We will use the same montage and parameters as active tDCS, but the active current will be applied for $30 \mathrm{~s}$ in the beginning and at the end of the procedure to simulate the same sensations of the current ramping as 


\section{Box 1 Inclusion and exclusion criteria}

\section{Inclusion criteria}

1. Age range $18-65$ years.

2. Diagnosis of fibromyalgia pain according to the American College of Rheumatology (ACR) 2010 criteria (existing pain for more than 6 months with an average of at least 4 on a $0-10$ Visual Analogue Scale (VAS) scale) without other comorbid chronic pain diagnosis.

3. Pain resistant to common analgesics and medications for chronic pain such as Tylenol, Aspirin, Ibuprofen, Soma, Parafon Forte DCS, Zanaflex and Codeine.

4. Must have the ability to feel sensation by Von-Frey fibre on the forearm.

5. Able to provide informed consent to participate in the study.

\section{Exclusion criteria}

1. Clinically significant or unstable medical or psychiatric disorder.

2. History of substance abuse within the past 6 months as self-reported (if subject reports a history of substance abuse, we will confirm using the Diagnostic and Statistical Manual of Mental Disorders, Fifth Edition (DSM V criteria).

3. Previous significant neurological history (eg, traumatic brain injury), resulting in neurological deficits, such as cognitive or motor deficits, as self-reported.

4. Previous neurosurgical procedure with craniotomy.

5. Severe depression (with a score of $>30$ on the Beck Depression Inventory).

6. Pregnancy -as the safety of tDCS in pregnant population (and children) has not been assessed (though the risk is non-significant), we will exclude pregnant women (and children). Women of childbearing age will be required to take a urine pregnancy test during the screening process and in every week of stimulation).

7. Current opiate use in large doses (more than $30 \mathrm{mg}$ of oxycodone/ hydrocodone or $7.5 \mathrm{mg}$ of hydromorphone (Dilaudid) or equivalent).

8. Patients will be excluded when they have increased risk for exercise defined as (i) not fulfilling the American College of Sports Medicine criteria (ie, risk of cardiovascular complication ${ }^{48}$ ) and in this case not cleared by a licensed physician.

in active stimulation. ${ }^{49}$ Using $30 \mathrm{~s}$ of ramping is reliable for blinding ${ }^{50}$ and less than 3 min of tDCS induces no cortical excitability effects. ${ }^{49}$
A tDCS adverse events questionnaire will be administered after each stimulation session. Subjects will be instructed not to use other methods of electrical stimulation during the trial.

\section{Outcomes}

Evaluation of endogenous pain inhibition system (primary outcomes)

During the CPM and TSPS protocols, heat pulses will be generated by a TSA-II Stimulator (Medoc Advanced Medical Systems, Ramat Yishai, Israel) delivered to the right proximal volar forearm using a $30 \mathrm{~mm} \times 30$ $\mathrm{mm}$ embedded heat pain (HP) thermode. A minimum interval of 10 min between the two assessments will be respected.

Conditioned pain modulation (CPM) evaluates the ability to inhibit pain. When a pain test stimulus is given together with a conditioning pain stimulus, the test stimulus is perceived as less painful than when it was given alone. ${ }^{51}$ We will follow the adapted protocol suggested by Granot et al $(2008)^{52}$ and Nir et al (2011).$^{53}$ We will first determine the pain-60 test temperature (which is the temperature that induces pain sensation at a magnitude of 60 on a 60-100 numerical pain scale (NPS)) by applying a Peltier thermode (Medoc Advanced Medical Systems, Ramat Yishai, Israel) on the right forearm and delivering three short heat stimuli $\left(43^{\circ} \mathrm{C}, 44^{\circ} \mathrm{C}\right.$ and $\left.45^{\circ} \mathrm{C}\right)$, each lasting 7 $\mathrm{s}$ (starting from the time the stimulus intensity reaches the destination temperature). Subjects will be asked to rate the level of pain intensity using a NPS ranging from $0=$ 'no pain' to $100=$ 'the worst pain imaginable'. If the first temperature of $43^{\circ} \mathrm{C}$ is considered too painful $(>60 / 100)$, we will stop the series and will provide additional stimuli at lower temperatures of $41^{\circ} \mathrm{C}$ and $42^{\circ} \mathrm{C}$. If the three temperatures $\left(43^{\circ} \mathrm{C}, 44^{\circ} \mathrm{C}\right.$ and $\left.45^{\circ} \mathrm{C}\right)$ are unable to achieve pain-60, we will deliver additional stimuli at $46^{\circ} \mathrm{C}$, $47^{\circ} \mathrm{C}$ and $48^{\circ} \mathrm{C}$ until reaching the desired pain level of $60 / 100$; in the unlikely event that none of those temperatures elicits pain-60, we will consider it to be $48^{\circ} \mathrm{C}$. On determining the pain-60 temperature, we will administer the test stimulus at that temperature for $30 \mathrm{~s}$, and subjects

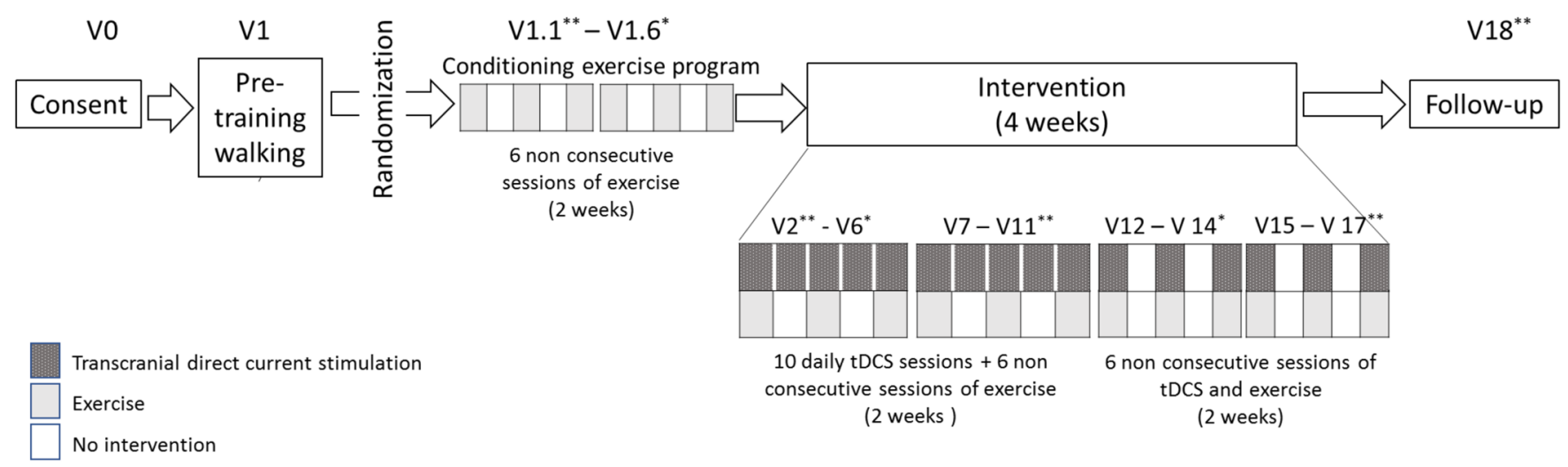

Figure 2 Schematic view of the timeline. tDCS, transcranial direct current stimulation. 
will be asked to rate their pain intensity at 10, 20 and 30 $\mathrm{s}$ after the thermode reaches the pain-60 temperature (mean scores of the three pain ratings will be calculated). Five minutes after delivering the test stimulus, the conditioning stimulus will be applied: the subject's left hand will be immersed for $30 \mathrm{~s}$ in a water bath set at $10^{\circ} \mathrm{C}-12^{\circ} \mathrm{C}$. Then, the same pain-60 temperature will be applied to the right forearm (left hand will still be immersed) for $30 \mathrm{~s}$ and the subject will again be asked to rate their pain intensity three times after the thermode reaches the pain-60 temperature: at 10, 20 and $30 \mathrm{~s}$ (mean scores of the three pain ratings will be calculated). CPM response will be calculated as the difference between the average of pain ratings from the test stimulus minus the average of pain ratings during the conditioned stimulus.

Temporal slow pain summation (TSPS) represents summation of $\mathrm{C}$ fibre mediated pain, assesses central sensitivity and is used to probe pain processing abnormalities in several chronic pain disorders. ${ }^{545}$ Subjects will be trained to identify pain-60 test temperature (see CPM protocol above) and we will follow the adapted protocol suggested by Staud et al $(2014)^{56}$ in which the HP-thermode was programmed to deliver pulses with rise/fall of 1-2 s, depending on subject's pain-60 level, from adapting temperatures to peak temperatures, with a plateau of 0.7 s. They will receive 1 train of 15 repetitive heat stimuli at $0.4 \mathrm{~Hz}$, which (being suitable to elicit TSPS in most subjects) allows the rating of individual pain stimuli and is unlikely to induce peripheral sensitisation. ${ }^{57}$ TSPS will be calculated as the difference between HP rating after the 1 st and 15th stimuli.

\section{Evaluation of cortical markers of inhibitory control (secondary neurophysiological outcomes) Transcranial magnetic stimulation (TMS)}

To assess tDCS and AE effects, we will measure the excitability of pain-related pathways using TMS markers. TMS assessments will be similar to our previous study. ${ }^{58}$ Single pulse TMS will be performed to acquire resting motor threshold (rMT) and motor evoked potentials (MEPs); paired pulse technique will measure short interval cortical inhibition (SICI) and intracortical facilitation (ICF). We will use Magstim Rapid ${ }^{2}$ device with a figureof-eight magnetic stimulator coil placed on the right and left M1 (for all assessments) and will record surface electromyogram from the contralateral first dorsal interosseous muscle. TMS data will be recorded and stored in a computer for off-line analysis.

1. Resting motor threshold ( $r M T)$ : Initially, we will investigate rMT following the technique described by Rossini and colleagues, where rMT is defined as the lowest stimulus intensity to evoke a MEP of $100 \mu \mathrm{V}$ in $3 / 5$ trials in the relaxed muscle. ${ }^{59}$

2. Motor evoked potential: We will initially adjust TMS machine output intensity to achieve a baseline MEP of 1 $\mathrm{mV}$ peak-to-peak amplitude before the intervention. Stimulation intensity will be kept constant for each subject throughout the evaluation sessions. We will re- cord 10 MEPs for each assessment and average their peak-to-peak amplitudes and areas-under the-curve.

3. Short interval intracortical inhibition (SICI) and intracortical facilitation (ICF): We will use paired pulse testing with a subthreshold conditioning stimulus $(80 \%$ rMT) followed by a suprathreshold test stimulus of $120 \%$ of the motor threshold. Interstimulus intervals will be 2 $\mathrm{ms}$ for SICI and $10 \mathrm{~ms}$ for ICF. Ten randomised stimuli will be applied at each interval and the percentage of inhibition or facilitation for each interstimulus interval before and after treatment will be calculated. The paired pulse MEP intensity will be the machine output intensity eliciting $1 \mathrm{mV}$ peak-to-peak amplitude that day-not the baseline MEP intensity used for single pulse testing. If we cannot obtain rMT, we will not perform MEPs or paired pulse.

\section{Electroencephalography (EEG)}

EEG will take place over approximately $45 \mathrm{~min}$ : $25 \mathrm{~min}$ of participant and software preparation, 10 min of EEG recording divided into a resting EEG condition (5 min with eyes open, 5 min with eyes closed) and a task-related condition (8 min). Participants will be asked to relax in the resting condition; the investigator will ensure they do not fall asleep.

The task-related condition will include movement observation (MO), movement imagery (MI) and movement execution (ME). This will be recorded by connecting the Net Station software (for EGI) with E-Prime. The entire task-related condition part will consist of 60 trials, with 20 trials for each of MO, MI and ME in a randomised order. ${ }^{60} 61$ Each trial will involve initial fixation (on a cross on a screen), followed by a visual cue stating the task to be performed ('imagine' and 'clench'), and a video will automatically play for observation. During each MO trial, the participant will view a video of a right hand clenching; during the MI task, the participant will be asked to imagine clenching her/his right hand once, and during the ME task, the subject will be asked to clench her/his right hand once. There will be a $4 \mathrm{~s}$ rest period between each trial. The purpose of the task-related condition is to evaluate ERD that reflects the motor cortex activation. ${ }^{62}$

We will record the EEG in a standardised way ${ }^{63}$ using the 64-channel EGI system (EGI, Eugene, USA). The EEG will be recorded with a band-pass filter of 0.3-200 $\mathrm{Hz}$ and digitised at the sampling rate of $250 \mathrm{~Hz}^{64}$ by connecting the Net Station software (for EGI) with E-Prime. On acquiring the EEG data, the EEGs will be inspected and artefacts will be cleaned manually. We will use EEGLAB and analysis of EEG data will include a power analysis of the power bands in the resting EEG portion-delta $(1-4 \mathrm{~Hz})$, theta $(4-8 \mathrm{~Hz})$, alpha $(8-13 \mathrm{~Hz})$ and beta $(13-30 \mathrm{~Hz})$ bands-fast Fourier transformation, Independent Component Analysis (ICA) decomposition, ERD responses of the three different motor tasks, functional connectivity measures and topographical analysis. The analysis will compare groups at baseline, during the 


\begin{tabular}{|c|c|c|c|}
\hline Study & Population & Intervention & Effect size \\
\hline \multicolumn{4}{|c|}{ Scenario I: tDCS effect on CPM in chronic pain } \\
\hline Ribeiro et al $(2017)^{72}$ & $\begin{array}{l}40 \text { women with chronic pain } \\
\text { undergoing hallux valgus surgery }\end{array}$ & Active versus sham tDCS & Cohen's $d=0.79$ \\
\hline \multicolumn{4}{|c|}{ Scenario II: tDCS effect on CPM in healthy volunteers } \\
\hline Braulio et al $(2018)^{73}$ & 48 healthy males & $\begin{array}{l}\text { Active-tDCS+remifentanil versus } \\
\text { sham-tDCS+remifentanil }\end{array}$ & Cohen's $d=0.98$ \\
\hline Flood et al $(2017)^{74}$ & 12 healthy males & $\begin{array}{l}\text { Active High-Definition } \\
\text { transcranial Direct Current } \\
\text { Stimulation (HD-tDCS) versus } \\
\text { sham HD-tDCS }\end{array}$ & Cohen's d=1.38 \\
\hline Flood et al (2016) ${ }^{75}$ & 30 healthy males & Active versus sham tDCS & Cohen's $d=0.89$ \\
\hline da Silva et al (2015) ${ }^{76}$ & 20 healthy males & $\begin{array}{l}\text { Active tDCS+melatonin versus } \\
\text { placebo+sham-tDCS }\end{array}$ & Cohen's $d=0.67$ \\
\hline Pooled effect size & & & 1.02 \\
\hline \multicolumn{4}{|c|}{ Scenario III: Exercise effect on CPM in chronic pain } \\
\hline Meeus et al (2015) ${ }^{77}$ & 16 rheumatoid arthritis & Exercise pre and post & Cohen's $d=0.78$ \\
\hline
\end{tabular}

CPM, conditioned pain modulation; tDCS, transcranial direct current stimulation.

stimulation period, on the last day of the intervention and at the 3 months follow-up.

\section{Secondary clinical outcomes}

The following secondary outcomes will be assessed: average pain intensity as assessed by Modified Brief Pain Inventory; Revised Fibromyalgia Impact Questionnaire; quality of life assessed by Quality of Life Scale, Patient Reported Outcomes Measurement Information System; Pittsburgh Sleep Quality Index and Beck Depression Inventory.

\section{Timeline}

This trial has 25 visits divided into four components (consent and pretraining walking, conditioning exercise programme, intervention and follow-up). To increase adherence to protocol, we will adjust the calendar of sessions according to the subject's availability (figure 2).

\section{Study sample}

Our target population is individuals with FM according to the ACR 2010 criteria. We plan to enrol 148 subjects divided into 4 groups ( $\mathrm{n}=37$ /group).

\section{Sample size calculation}

We used the information from trials measuring the effects of tDCS and AE on CPM and TSPS according to different scenarios to do this sample size calculation (table 1).

- In Scenario I, we considered the effects of tDCS on CPM in patients with chronic pain: this resulted in an effect size (ES) of 0.79 .

- In Scenario II, we evaluated the effect of tDCS on CPM in healthy volunteers: this resulted in a pooled ES of 1.02.
- In Scenario III, we evaluated the effect of exercise on CPM in chronic pain and this resulted in an ES of 0.78 .

Based on this analysis, we decided on a conservative approach and chose the lowest ES; thus, we used an ES of 0.78 . In addition, it is important to underscore that we expect that the combination of $\mathrm{tDCS}+\mathrm{AE}$ will have a higher effect than each intervention alone (tDCS, exercise or placebo). Additionally, in this current proposal, the dosage of tDCS is higher than the studies we used to calculate the sample size (see tables 1 and 2).

We assumed a type I error of $5 \%$ (alpha) and made a sensitivity analysis with a type 2 error (beta) of $10 \%, 15 \%$ and $20 \%$ (therefore a power of $90 \%, 85 \%$ and $80 \%$ ). We used a t-test for two independent means and considered dropout rates of $20 \%$ and $15 \%$ (table 2).

Although most studies used a power of $80 \%$ and a dropout rate of $10 \%-15 \%,{ }^{22} 2965-70$ we chose a dropout

Table 2 Two-tailed analyses

\begin{tabular}{lllll}
\hline & $\begin{array}{l}\text { Alpha } \\
\text { (\%) }\end{array}$ & ES & $\begin{array}{l}\text { Dropout } \\
\text { rate (\%) }\end{array}$ & $\begin{array}{l}\text { Final total } \\
\text { sample size } \\
\text { (four groups) }\end{array}$ \\
\hline Power of $80 \%$ & 5 & 0.78 & 15 & 124 \\
\hline Power of $85 \%$ & 5 & 0.78 & 15 & 142 \\
\hline Power of $90 \%$ & 5 & 0.78 & 15 & 165 \\
\hline Power of $80 \%$ & 5 & 0.78 & 20 & 130 \\
\hline Power of $85 \%$ & 5 & 0.78 & 20 & 148 \\
\hline Power of $90 \%$ & 5 & 0.78 & 20 & 172 \\
\hline
\end{tabular}

ES, effect size. 
rate of $20 \%$ and power of $85 \%$ as to be more conservative and also account for unexpected factors.

\section{Data analysis}

All data collected will be kept in a secured and password protected database, accessible only to IRB trained and approved study staff. All analyses will be performed as intention-to-treat in which all randomised subjects who receive at least one intervention session will be included. We will conduct sensitivity analyses and test different models of handling missing data: Last Observation Carried Forward and Multiple Imputation. The change in the primary efficacy endpoints, CPM and TSPS, from baseline to week 4 , will be tested with a mixed linear regression model. This model will be adjusted for important demographic variables (eg, gender) and baseline clinical parameters where appropriate. All tests will be two-sided (alpha level 0.05).

We will initially test our main hypothesis that active tDCS+AE increases CPM and decreases TSPS more than sham tDCS+nAE. If the effect is significant, we will then test differences between the active tDCS+AE group versus the two interventions alone. We will run a secondary mixed linear model to estimate the rate of change over time (using the secondary endpoints added in this model-Week 2 and follow-up) and also include the interaction term (treatment*time) to detect whether treatment effect changes differently over time. If the interaction is not significant, we will then test whether there is a main effect of time that is independent of treatment level (interaction will be removed from the model). We will adjust this model for important covariates such as age, gender, pain levels (NPS) and other baseline clinical outcomes where appropriate. For secondary clinical variables with significant effects, we will test whether they moderate the interventions' effects on our mechanistic (TMS and EEG) outcomes, thereby gaining additional mechanistic insights. To complete our analysis, we will apply a path analysis ${ }^{71}$ to CPM and TSPS to determine if endogenous pain modulation changes (indexed by CPM and TSPS) associated with active tDCS+AE is related to direct effects versus indirect effects through secondary outcome improvements. We propose that a direct effect of active tDCS and $\mathrm{AE}$ on the endogenous pain inhibitory system can be inferred if the treatment effect cannot be explained by changes in psychological or functional outcomes.

An independent monitoring committee will review data on recruitment, adherence and safety; meetings will occur annually, after enrolment of $25 \%$ of the target sample or in case of reports of any serious adverse events. NIH will also perform annual site monitoring visits.

\section{Patient and public involvement}

Patients and public were not involved in the design of this study.

\section{Ethics and dissemination}

This protocol was approved by the IRB at the Partners Human Research Committee (Protocol approval number: 2017P002524). All requirements regarding the welfare, rights and privacy of human subjects protection were fulfilled. The risks of this clinical trials were considered to be minimal and are addressed in the protocol and consent form. Informed consent will be obtained from all participants before any study procedures by the Principal Investigator or coinvestigators. Trial registration number: NCT03371225. For a complete list of trial registration dataset and protocol version history, please refer to online supplementary file 1 .

The study findings will be reported in conferences and in peer-reviewed journal publications.

\section{Author affiliations}

${ }^{1}$ Neuromodulation Center/Spaulding Rehabilitation Hospital, Harvard Medical School, Boston, Massachusetts, USA

${ }^{2}$ Laboratory of Pain \& Neuromodulation, Hospital de Clinicas de Porto Alegre da Universidade Federal do Rio Grande do Sul, Porto Alegre, Brazil

Contributors LC-B and EUK are joint first authors and with FF conceptualised the paper. PG-M, LC-S and AE-G wrote the abstract. LC-B, EUK, LC-S, ME-G wrote the introduction. AC-R, CBP, IM-T, EUK, LC-B, MME, KP-B, PG-M, DD, AE-G and YY wrote methods and analysis. AC-R, IM-T and YY prepared the figures. FF, MME, WC and HR provided critical review.

Funding This work is supported by NIH grant R01 AT009491-01A1.

Competing interests None declared.

Patient consent for publication Not required.

Ethics approval This study obeys the Declaration of Helsinki and was approved by the Institutional Review Board (IRB) of Partners Healthcare under the protocol number 2017P002524.

Provenance and peer review Not commissioned; externally peer reviewed.

Open access This is an open access article distributed in accordance with the Creative Commons Attribution Non Commercial (CC BY-NC 4.0) license, which permits others to distribute, remix, adapt, build upon this work non-commercially, and license their derivative works on different terms, provided the original work is properly cited, appropriate credit is given, any changes made indicated, and the use is non-commercial. See: http://creativecommons.org/licenses/by-nc/4.0/.

ORCID iDs

Elif Uygur Kucukseymen http://orcid.org/0000-0002-6190-106X

Alejandra Cardenas-Rojas http://orcid.org/0000-0002-8464-6866

Paola Gonzalez-Mego http://orcid.org/0000-0002-2382-2061

\section{REFERENCES}

1. Wolfe F, Brähler E, Hinz A, et al. Fibromyalgia prevalence, somatic symptom reporting, and the dimensionality of polysymptomatic distress: results from a survey of the general population. Arthritis Care Res 2013;65:777-85.

2. Verbunt JA, Pernot DHFM, Smeets RJEM. Disability and quality of life in patients with fibromyalgia. Health Qual Life Outcomes 2008;6:8.

3. Robinson RL, Birnbaum HG, Morley MA, et al. Economic cost and epidemiological characteristics of patients with fibromyalgia claims. $J$ Rheumatol 2003;30:1318-25.

4. Adams N, Sim J. Rehabilitation approaches in fibromyalgia. Disabil Rehabil 2005;27:711-23.

5. Häuser W, Walitt B, Fitzcharles M-A, et al. Review of pharmacological therapies in fibromyalgia syndrome. Arthritis Res Ther 2014;16.

6. Basbaum Al, Fields HL. Endogenous pain control systems: brainstem spinal pathways and endorphin circuitry. Annu Rev Neurosci 1984;7:309-38. 
7. Ossipov MH, Dussor GO, Porreca F. Central modulation of pain. $J$ Clin Invest 2010;120:3779-87.

8. Ossipov MH, Morimura K, Porreca F. Descending pain modulation and chronification of pain. Curr Opin Support Palliat Care 2014;8:143.

9. Staud R. Abnormal endogenous pain modulation is a shared characteristic of many chronic pain conditions. Expert Rev Neurother 2012;12:577-85.

10. de la Coba P, Bruehl S, Moreno-Padilla M, et al. Responses to slowly repeated evoked pain stimuli in fibromyalgia patients: evidence of enhanced pain sensitization. Pain Med 2017;18:1778-86.

11. Julien N, Goffaux P, Arsenault P, et al. Widespread pain in fibromyalgia is related to a deficit of endogenous pain inhibition. Pain 2005;114:295-302.

12. Mhalla A, de Andrade DC, Baudic S, et al. Alteration of cortical excitability in patients with fibromyalgia. Pain 2010;149:495-500.

13. Gibson SJ, Littlejohn GO, Gorman MM, et al. Altered heat pain thresholds and cerebral event-related potentials following painful $\mathrm{CO} 2$ laser stimulation in subjects with fibromyalgia syndrome. Pain 1994:58:185-93.

14. Stevens A, Batra A, Kötter I, et al. Both pain and EEG response to cold pressor stimulation occurs faster in fibromyalgia patients than in control subjects. Psychiatry Res 2000;97:237-47.

15. Cardinal TM, Antunes LC, Brietzke AP, et al. Differential neuroplastic changes in fibromyalgia and depression indexed by up-regulation of motor cortex inhibition and disinhibition of the descending pain system: an exploratory study. Front Hum Neurosci 2019;13:138.

16. Deitos A, Soldatelli MD, Dussán-Sarria JA, et al. Novel insights of effects of pregabalin on neural mechanisms of intracortical disinhibition in physiopathology of fibromyalgia: an explanatory, randomized, double-blind crossover study. Front Hum Neurosci 2018;12.

17. Pujol J, López-Solà M, Ortiz H, et al. Mapping brain response to pain in fibromyalgia patients using temporal analysis of fMRI. PLoS One 2009;4:e5224.

18. Williams DA, Gracely RH. Biology and therapy of fibromyalgia. functional magnetic resonance imaging findings in fibromyalgia. Arthritis Res Ther 2006;8.

19. Burgmer M, Pogatzki-Zahn E, Gaubitz M, et al. Altered brain activity during pain processing in fibromyalgia. Neuroimage 2009;44:502-8.

20. Schrepf A, Harper DE, Harte SE, et al. Endogenous opioidergic dysregulation of pain in fibromyalgia: a PET and fMRI study. Pain 2016;157:2217.

21. Gracely RH, Petzke F, Wolf JM, et al. Functional magnetic resonance imaging evidence of augmented pain processing in fibromyalgia. Arthritis Rheum 2002;46:1333-43.

22. Busch AJ, Barber KAR, Overend TJ, et al. Exercise for treating fibromyalgia syndrome. Cochrane Database Syst Rev 2007;24.

23. Fregni F, Boggio PS, Lima MC, et al. A sham-controlled, phase II trial of transcranial direct current stimulation for the treatment of central pain in traumatic spinal cord injury. Pain 2006;122:197-209.

24. Dasilva AF, Mendonca ME, Zaghi S, et al. tDCS-induced analgesia and electrical fields in pain-related neural networks in chronic migraine. Headache 2012;52:1283-95.

25. Antal A, Terney D, Kühnl S, et al. Anodal transcranial direct current stimulation of the motor cortex ameliorates chronic pain and reduces short intracortical inhibition. J Pain Symptom Manage 2010;39:890-903.

26. Fenton BW, Palmieri PA, Boggio P, et al. A preliminary study of transcranial direct current stimulation for the treatment of refractory chronic pelvic pain. Brain Stimul 2009;2:103-7.

27. Vaseghi B, Zoghi M, Jaberzadeh S. Does anodal transcranial direct current stimulation modulate sensory perception and pain? A metaanalysis study. Clin Neurophysiol 2014;125:1847-58.

28. Fregni F, Freedman S, Pascual-Leone A. Recent advances in the treatment of chronic pain with non-invasive brain stimulation techniques. Lancet Neurol 2007;6:188-91.

29. O'Connell NE, Marston L, Spencer S, et al. Non-Invasive brain stimulation techniques for chronic pain. Cochrane Database Syst Rev 2018;3.

30. Zhu C-E, Yu B, Zhang W, et al. Effiectiveness and safety of transcranial direct current stimulation in fibromyalgia: a systematic review and meta-analysis. J Rehabil Med 2017;49:2-9.

31. Fregni F, Gimenes R, Valle AC, et al. A randomized, shamcontrolled, proof of principle study of transcranial direct current stimulation for the treatment of pain in fibromyalgia. Arthritis Rheum 2006;54:3988-98.

32. Fagerlund AJ, Hansen OA, Aslaksen PM. Transcranial direct current stimulation as a treatment for patients with fibromyalgia: a randomized controlled trial. Pain 2015;156:62-71.
33. Carvalho F, Brietzke AP, Gasparin A, et al. Home-Based transcranial direct current stimulation device development: an updated protocol used at home in healthy subjects and fibromyalgia patients. JoVE 2018:137:e57614.

34. Brunoni AR, Valiengo L, Baccaro A, et al. The sertraline vs. electrical current therapy for treating depression clinical study: results from a factorial, randomized, controlled trial. JAMA Psychiatry 2013;70:383-91.

35. Hazime FA, Baptista AF, de Freitas DG, et al. Treating low back pain with combined cerebral and peripheral electrical stimulation: a randomized, double-blind, factorial clinical trial. Eur J Pain 2017;21:1132-43.

36. Viana RT, Laurentino GEC, Souza RJP, et al. Effects of the addition of transcranial direct current stimulation to virtual reality therapy after stroke: a pilot randomized controlled trial. NeuroRehabilitation 2014;34:437-46.

37. Mendonca ME, Simis M, Grecco LC, et al. Transcranial direct current stimulation combined with aerobic exercise to optimize analgesic responses in fibromyalgia: a randomized placebo-controlled clinical trial. Front Hum Neurosci 2016;10:68.

38. Ellingson LD, Stegner AJ, Schwabacher IJ, et al. Exercise strengthens central nervous system modulation of pain in fibromyalgia. Brain Sci 2016;6. doi:10.3390/brainsci6010008.

39. Theadom A, Cropley M, Smith HE, et al. Mind and body therapy for fibromyalgia. Cochrane Database Syst Rev 2015;13.

40. Castillo-Saavedra L, Gebodh N, Bikson M, et al. Clinically effective treatment of fibromyalgia pain with high-definition transcranial direct current stimulation: phase II open-label dose optimization. J Pain 2016;17:14-26.

41. Villamar MF, Wivatvongvana P, Patumanond J, et al. Focal modulation of the primary motor cortex in fibromyalgia using $4 \times 1$ ring high-definition transcranial direct current stimulation (HD-tDCS): immediate and delayed analgesic effects of cathodal and anodal stimulation. J Pain 2013;14:371-83.

42. Borckardt JJ, Bikson M, Frohman $\mathrm{H}$, et al. A pilot study of the tolerability and effects of high-definition transcranial direct current stimulation (HD-tDCS) on pain perception. J Pain 2012;13:112-20.

43. Stephens S, Feldman BM, Bradley N, et al. Feasibility and effectiveness of an aerobic exercise program in children with fibromyalgia: results of a randomized controlled pilot trial. Arthritis Rheum 2008;59:1399-406.

44. Oliveira NC, dos Santos Sabbag LM, de Sá Pinto AL, et al. Aerobic exercise is safe and effective in systemic sclerosis. Int J Sports Med 2009;30:728-32.

45. Gill TM, DiPietro L, Krumholz HM. Role of exercise stress testing and safety monitoring for older persons starting an exercise program. JAMA 2000;284:342-9.

46. Roveda F, Middlekauff HR, Rondon MUPB, et al. The effects of exercise training on sympathetic neural activation in advanced heart failure: a randomized controlled trial. J Am Coll Cardiol 2003;42:854-60.

47. Geneen LJ, Moore RA, Clarke C, et al. Physical activity and exercise for chronic pain in adults: an overview of Cochrane reviews. Cochrane Database Syst Rev 2017;4.

48. ACSM. ACSM's Guidelines for Exercise Testing and Prescription. In: Williams L, ed. 9Th, 2015.

49. Nitsche MA, Paulus W. Excitability changes induced in the human motor cortex by weak transcranial direct current stimulation. $J$ Physiol 2000;527 Pt 3:633-9.

50. Gandiga PC, Hummel FC, Cohen LG. Transcranial DC stimulation (tDCS): a tool for double-blind sham-controlled clinical studies in brain stimulation. Clin Neurophysiol 2006;117:845-50.

51. Potvin S, Marchand S. Pain facilitation and pain inhibition during conditioned pain modulation in fibromyalgia and in healthy controls. Pain 2016;157:1704-10.

52. Granot M, Weissman-Fogel I, Crispel Y, et al. Determinants of endogenous analgesia magnitude in a diffuse noxious inhibitory control (DNIC) paradigm: do conditioning stimulus painfulness, gender and personality variables matter? Pain 2008;136:142-9.

53. Nir R-R, Granovsky Y, Yarnitsky D, et al. A psychophysical study of endogenous analgesia: the role of the conditioning pain in the induction and magnitude of conditioned pain modulation. Eur J Pain 2011;15:491-7.

54. Bosma RL, Mojarad EA, Leung L, et al. Fmri of spinal and supraspinal correlates of temporal pain summation in fibromyalgia patients. Hum Brain Mapp 2016;37:1349-60.

55. Craggs JG, Staud R, Robinson ME, et al. Effective connectivity among brain regions associated with slow temporal summation of C-fiber-evoked pain in fibromyalgia patients and healthy controls. $J$ Pain 2012;13:390-400. 
56. Staud R, Weyl EE, Riley JL, et al. Slow temporal summation of pain for assessment of central pain sensitivity and clinical pain of fibromyalgia patients. PLoS One 2014;9:e89086.

57. Vierck CJ, Cannon RL, Fry G, et al. Characteristics of temporal summation of second pain sensations elicited by brief contact of glabrous skin by a preheated thermode. $J$ Neurophysiol 1997;78:992-1002.

58. Williams JA, Pascual-Leone A, Fregni F. Interhemispheric modulation induced by cortical stimulation and motor training. Phys Ther 2010;90:398-410.

59. Rossini PM, Micera S, Benvenuto A, et al. Double nerve intraneural interface implant on a human amputee for robotic hand control. Clin Neurophysiol 2010;121:777-83.

60. Li H, Huang G, Lin Q, et al. Combining movement-related cortical potentials and event-related desynchronization to study movement preparation and execution. Front Neurol 2018;9.

61. Duann J-R, Chiou J-C. A comparison of independent eventrelated desynchronization responses in motor-related brain areas to movement execution, movement imagery, and movement observation. PLoS One 2016;11:e0162546.

62. Neuper C, Wörtz M, Pfurtscheller G. ERD/ERS patterns reflecting sensorimotor activation and deactivation. Prog Brain Res 2006;159:211-22.

63. Nuwer MR, Lehmann D, Lopes da Silva F, et al. IFCN guidelines for topographic and frequency analysis of EEGs and Eps. Report of an IFCN Committee. Electroencephalogr Clin Neurophysiol 1994;91:1-5.

64. Liu C, Wang $\mathrm{H}, \mathrm{Pu} \mathrm{H}$, et al. EEG feature extraction and pattern recognition during right and left hands motor imagery in braincomputer interface. In: 2012 5th International Conference on biomedical engineering and informatics, 2012.

65. García-Hermoso A, Saavedra JM, Escalante Y. Effects of exercise on functional aerobic capacity in adults with fibromyalgia syndrome: a systematic review of randomized controlled trials. J Back Musculoskelet Rehabil 2015;28:609-19.

66. Bidonde J, Busch AJ, Schachter CL, et al. Aerobic exercise training for adults with fibromyalgia. Cochrane Database Syst Rev 2017;15.

67. Wang $\mathrm{C}$, Schmid $\mathrm{CH}$, Fielding RA, et al. Effect of tai chi versus aerobic exercise for fibromyalgia: comparative effectiveness randomized controlled trial. $B M J$ 2018;360.
68. Marske C, Bernard N, Palacios A, et al. Fibromyalgia with gabapentin and osteopathic manipulative medicine: a pilot study. J Altern Complement Med 2018;24:395-402.

69. Fontaine KR, Conn L, Clauw DJ. Effects of lifestyle physical activity on perceived symptoms and physical function in adults with fibromyalgia: results of a randomized trial. Arthritis Res Ther 2010;12.

70. Valle A, Roizenblatt S, Botte S, et al. Efficacy of anodal transcranial direct current stimulation (tDCS) for the treatment of fibromyalgia: results of a randomized, sham-controlled longitudinal clinical trial. $J$ Pain Manag 2009;2:353-61.

71. Möller HJ, Müller H, Borison RL, et al. A path-analytical approach to differentiate between direct and indirect drug effects on negative symptoms in schizophrenic patients. A re-evaluation of the North American risperidone study. Eur Arch Psychiatry Clin Neurosci 1995;245:45-9.

72. Ribeiro H, Sesterhenn RB, Souza Ade, et al. Preoperative transcranial direct current stimulation: exploration of a novel strategy to enhance neuroplasticity before surgery to control postoperative pain. A randomized sham-controlled study. PLoS One 2017; 12:e0187013.

73. Braulio G, Passos SC, Leite F, et al. Effects of transcranial direct current stimulation block Remifentanil-Induced hyperalgesia: a randomized, double-blind clinical trial. Front Pharmacol 2018;9:94.

74. Flood A, Waddington G, Keegan RJ, et al. The effects of elevated pain inhibition on endurance exercise performance. PeerJ 2017;5:e3028.

75. Flood A, Waddington G, Cathcart S. High-Definition Transcranial Direct Current Stimulation Enhances Conditioned Pain Modulation in Healthy Volunteers: A Randomized Trial. J Pain 2016;17:600-5.

76. da Silva NRJ, Laste G, Deitos A, et al. Combined neuromodulatory interventions in acute experimental pain: assessment of melatonin and non-invasive brain stimulation. Front Behav Neurosci 2015;9:77.

77. Meeus M, Hermans L, Ickmans K, et al. Endogenous pain modulation in response to exercise in patients with rheumatoid arthritis, patients with chronic fatigue syndrome and comorbid fibromyalgia, and healthy controls: a double-blind randomized controlled trial. Pain Pract 2015;15:98-106. 